\title{
Alcohol dependence: provisional description of a clinical syndrome
}

\author{
GRIFFITH EDWARDS, MILTON M GROSS
}

British Medical fournal, 1976, 1, 1058-1061

Anyone concerned with treating drinking problems must find that his patients often tell him more than is in the textbooks. Each tells a different story, but there are also repeated patterns. Much of the varied experience that is recounted can be interpreted as the patient's astute observation of the alcohol dependence syndrome-a condition certainly far better described by the average alcoholic than in any book.

To attempt a definitive description of this syndrome would be premature; much is still only at the stage of "clinical impression." Routine clinical questions may impose a pattern on patients' accounts, and patients may themselves organise their uncertain recall of events in terms of expectations given to them. To link the clinical syndrome with information on the psychobiological basis of dependence is difficult, though scientific understanding has advanced recently. Our aim here is to help further to delineate the clinical picture.

This is far from the first attempt to describe the syndrome. Jellinek's classification of alcoholism into types stands supreme. ${ }^{1}$ The American National Council on Alcoholism has recently analysed diagnostic criteria, ${ }^{2}$ and a World Health Organisation group is preparing a report that seeks to define this syndrome and examine its importance.* Furthermore, we take the term syndrome to mean no more than the concurrence of phenomena. Not all the elements need always be present, nor always present with the same intensity. No assumptions need be made about

*WHO Group of Investigators on Criteria for Identifying and Classifying Disabilities Related to Alcohol Consumption.

Addiction Research Unit, Institute of Psychiatry, London SE5 8AF GRIFFITH EDWARDS, DM, Reader in drug dependence

Downstate Medical Centre, Brooklyn, New York 11203, USA MILTON M GROSS, MD, professor of psychiatry the cause or the pathological process, though the obvious scientific challenge is to understand the underlying reasons for the clustering and covariance.

\section{Essential elements of the syndrome}

Essential elements might provisionally include: a narrowing in the repertoire of drinking behaviour; salience of drink-seeking behaviour; increased tolerance to alcohol; repeated withdrawal symptoms; repeated relief or avoidance of withdrawal symptoms by further drinking; subjective awareness of a compulsion to drink; reinstatement of the syndrome after abstinence. All these elements exist in degree, thus giving the syndrome a range of severity. They represent the dimensions along which the clinician can order the information given to him; one clinical element may reflect underlying psychobiological happenings of several types, and different clinical elements may be partial descriptions of the same underlying psychobiological process. In discussing the clinical presentations of each element we shall give particular attention to degrees of possible development and to patterning in presentation by personal and social factors. ${ }^{3}$

\section{NARROWING OF THE DRINKING REPERTOIRE}

The ordinary drinker's consumption and beverage will vary from day to day and from week to week: he may have beer at lunch on one day, nothing to drink on another, share a bottle of wine at dinner one night, and then go to a party on a Saturday and have a lot to drink. His drinking is patterned by varying internal cues and external circumstances.

At first, a person becoming caught up in heavy drinking may often widen his repertoire and also the range of cues that signal drinking. As dependence advances, the cues are increasingly related to relief or avoidance of alcohol withdrawal and the personal drinking repertoire becomes increasingly narrowed. The dependent person begins to drink the same whether it is work day, weekend, or holiday: the nature of the company or his own mood makes less and less difference. Questioning may distinguish earlier and later stages of dependence by the degree to which the repertoire is narrowed. With advanced 
dependence the drinking may become stereotyped-scheduled to a daily time table to maintain a high blood alcohol-and the patient will be able to recount where and when each drink of the daily ration was bought and consumed. More careful questioning will, however, show that even when dependence is well established some capacity for variation remains. Change in personal circumstances such as a new job or a different marriage may for a time constrain the drinking. Pricing and sales regulations may also influence the dependent drinker. ${ }^{+}$The syndrome must be pictured as subtle and plastic rather than as something set hard, but as dependence advances the pattern tends to become increasingly stereotyped. Available data on the consumption of "alcoholics"s generally refer to heterogeneous samples with not all patients dependent or severely dependent and may underestimate the mean consumption of heavily dependent subjects.

\section{SALIENCE OF DRINK-SEEKING BEHAVIOUR}

This stereotyping of the drinking pattern as dependence advances leads to the individual giving priority to maintaining his alcohol intake; indeed the failure of unpleasant consequences to deter may be a clinical indicator of the degree of dependence. The wife's distressed scolding - once effective-is later neutralised by the drinker as evidence of her lack of understanding. Income which had previously to serve many needs now satisfies only the drive for drink. Gratification of the need for drink may become more important for the patient with liver damage than even considerations of survival-c"a short life and a merry one."

In clinical assessment attention has to be paid to the patient's basic personality, for many people are not dependent but none the less drink without much regard for consequences because of their general irresponsibility. These people may, of course, in the end develop the dependence syndrome. Diagnostically the progressive change in the salience given to alcohol is important rather than behaviour at any one time. Typically, the patient relates that he used to be proud of his house but now the paint is peeling off, used always to take the children to football matches but now spends no time with them, used to have rather rigid moral standards but will now beg, borrow, or steal to obtain money for alcohol. In the same way the drug-dependent monkey will work hard for its alcohol, ${ }^{6}$ and experiments with human volunteers have supported the appropriateness of such a model. ${ }^{7}$

\section{INCREASED TOLERANCE TO ALCOHOL}

Alcohol is a drug to which the central nervous system (CNS) develops tolerance. ${ }^{8}$ The precise mechanism is not yet known, but presumably there are changes at the synaptic junction ${ }^{9}-\mathrm{a}$ sort of homoeostatic adjustment to continued alcohol exposure. Metabolic tolerance (increased liver clearance) makes a relatively trivial contribution. Clinically, tolerance is shown by the dependent person being able to sustain an alcohol intake and go about his business at blood alcohol levels that would incapacitate the non-tolerant drinker. This does not mean his functions are unimpaired-he will be a dangerous driver-but because of his tolerance he will (unfortunately) still be able to drive. Cross-tolerance will extend to other general depressants such as barbiturates and minor tranquillisers. The rate of development of tolerance is still unknown, but the heavy drinker who is not dependent will probably also show considerable tolerance. In later stages of dependence, for reasons which are unclear, the individual begins to lose his previously acquired tolerance and then becomes incapacitated by quantities of alcohol which he could previously handle; for the first time he may fall down in the street.

Patients themselves report on tolerance as "having a good head for it" or "being able to drink the other man under the table." Questioning often reveals the patient's awareness that "just one or two drinks aren't any good"; he himself has sensed the crucial meaning of a change in the dose-response curve.

\section{REPEATED WITHDRAWAI SYMPTOMS}

Experiments on animals ${ }^{10}$ and men ${ }^{112}$ have amply confirmed the reality of an alcohol withdrawal syndrome. Patient's accounts vary with the degree of dependence. At first, symptoms are intermittent and mild and cause little incapacity, and one may be experienced without others. As dependence increases so do the frequency and the severity of the symptoms. When the picture is fully developed, typically the patient has severe multiple symptoms every morning on waking; they may wake him in the middle of the night. Questioning often shows that the severely dependent patient experiences mild withdrawal symptoms (which he recognises as such) at any time during the day when his alcohol level falls. Complete withdrawal is therefore not necessary to precipitate these symptoms. ${ }^{13}$

The bridge between experiment and clinical observation is incomplete in identifying the type of drinking in which the previously naive drinker must engage before withdrawal symptoms will be experienced. Clinically, it seems that to incur withdrawal symptoms an individual generally has to drink about 200-300 $\mathrm{g}$ of alcohol a day for several years - though there are many exceptions to that imprecise rule, and in either direction. Another unresolved problem is the timing of the first experience of withdrawal symptoms: the patient often remembers the moment precisely, and there is no necessary association with a sudden increase in alcohol intake. W'ithdrawal symptoms cannor occur without a high degree of CNS tolerance, but tolerance can exist without clinically manifest withdrawal symptoms.

The spectrum of symptoms is wide and includes tremor, nausea, sweating, hyperacusis, tinnitus, itching, muscle cramps, mood disturbance, sleep disturbance, perceptual distortion, hallucination, grand-mal seizures, and the fully developed picture of delirium tremens. ${ }^{14}$ There are four key symptoms.

Tremor nicely illustrates that it is range of experience that is the real stuff of the patient's report. It does violence to clinical reality to record in the case notes simply that the patient does or does not experience withdrawal shakes. Shakiness may have been experienced only once or twice or very intermittently and mildly or it may be experienced every morning and to a degree which is incapacitating, or with many intervening intensities and frequencies. As well as the hands shaking there may be an awareness of facial tremor or, indeed, of "the whole body shaking." The clinician has to cultivate an awareness of something equivalent to the Beaufort scale for wind strength and look for the patient's saying that he has given up drinking his morning tea from a cup (because it rattles against the saucer) and changed to a mug, that he has given up an ordinary razor for an electric razor, or that, in the extreme case, he is forced to rely on the kindness of the barmaid to lift the day's first pint to his lips.

Nausea-The patient who is simply asked whether he vomits may well deny it: his experience may be that if he attempts to clean his teeth in the morning he will make himself retch. He may never eat breakfast because he knows it would be too risky. A common story is that most of the first drink of the day is regularly vomited back. At the extreme, the patient may keep a bucket by his bed.

Sweating-This may be dramatic: the patient may wake regularly in the early hours of the morning with soaking sweats. At the earlier stages of dependence, on the other hand, he may report no more than feeling a bit clammy.

The mood disturbance of withdrawal is important in its own right and is not just a reaction to the physical distress. At the earlier stages of dependence patients may phrase the experience in terms of "being a bit edgy" or "nerves not too good," but when dependence is fully developed they may use vivid phrasing to indicate a state of appalling affective distress. Patients often find it hard to describe how they feel. Sometimes they may differentiate between anxiety and depression (though not necessarily using those words), and one patient may complain acutely of anxiety but of little depression, while another may complain of the opposite. Often the anxiety seems to be characterised by a frightened reaction to loud noises or traffic (sometimes with a phobia of crossing the road), a fear of a friend coming up suddenly from behind, a dislike of shadows at night, fright at "the twigs on the trees rubbing together." The oversensitivity can be like that of a gouty patient who fears a fly alighting on his toe.

RELIEF OR AVOIDANCE OF WITHDRAWAL SYMPTOMS BY FURTHER DRINKING

In the earliest stages the patient may just be aware that at lunchtime the first drink of the day "helps to straighten him up a bit." At the other extreme the patient may require a drink before he gets out of bed every morning as a matter of desperate need. As with withdrawal symptoms, relief drinking must not be conceived as only a morning event: the patient may wake in the middle of the night for the drink which will abort incipient withdrawal, and he may be well aware that if he has to go three or four hours without a drink during the day the next drink is valued especially for its relief effect. Relief drinking is probably cued not only by frank withdrawal but also by minimal symptoms of subacute withdrawal, which signal worse distress if drink is not then taken. The dependent individual may try to maintain a steady alcohol level which he has learnt to recognise as comfortably above the danger level for withdrawal, and to this extent his drinking is cued by withdrawal avoidance as well as withdrawal relief.

Clues to the degree of a patient's dependence are often given by the small details he provides on the circumstances and timing of the first drink of the day and his attitudes toward it. If he has time to get up and have a bath and dress and read the paper before the drink then 
dependence is not very advanced. A housewife who finishes her morning chores before having her first drink is at a different stage of dependence than the woman who is pouring whisky into her first cup of tea. Someone engaged in relief drinking may almost have ritualised the procedure. He will go to the early-morning market pub at $7 \mathrm{am}$, go straight up to the bar, and the barmaid will know immediately to give him his pint of cider, which he will grab at clumsily with both hands and drink down fast. He may go to the lavatory and vomit some of it back. But he can then drink a pint of beer at greater leisure, and he will know that within 20 or 30 minutes of walking into that pub "the drink will have cured him." The patient often relates that he knows the exact quantity of alcohol required for this cure and the exact time interval for the alcohol to take effect, and he reports also that the cure is repeatedly so complete as to be almost magical. Sometimes there is (presumably) a conditioned effect: the mere fact of having a glass in the hand may give some immediate relief.

That the dependence syndrome is a plastic condition rather than something immutable is brought out again by the shaping of this feature by social and personal factors. For the labourer the idea of keeping drink in the house may be so against subcultural expectations that he will always wait for the pubs to open (perhaps travelling specially to an early-morning market pub) rather than "keep a drink indoors." The man of rigid personality may endure considerable withdrawal for some hours rather than take a drink before lunch. Fully to understand what the patient reports always requires that these shaping factors are taken into account.

\section{SUBJECTIVE AWARENESS OF COMPULSION TO DRINK}

The conventional phrases used to describe the dependent person's subjective experience are not altogether satisfactory. For instance, awareness of "loss of control" is said to be crucial to understanding abnormal drinking, ${ }^{15}$ and patients do say "if I have one or two I'll go on," or "if I go into the pub promises don't mean anything," or "once I've really got the taste of it I'm away." It is unclear, however, whether the experience is truly one of losing control rather than one of deciding not to exercise control. Control is probably best seen as variably and intermittently impaired"16 rather than "lost." Another complex experience which can too easily be wrapped up in conventional phrasing is the experience of "craving." The patient may describe it in unambiguous terms-he may be "gasping for a drink." The subjective interpretation of the withdrawal may, however, be much influenced by environment, and the patient who is withdrawing on a ward may not experience any particular craving. Cues for craving may include the feeling of intoxication as well as incipient or developed withdrawal, affective states (anger, depression, elation), or situation cues (being in a pub or with a particular friend).

Perhaps the key experience can best be described as a compulsion to drink, and, though the analogy between alcohol dependence and compulsive disorder has not been considered very satisfactory in the past, the subjective experience of dependence may come close to fulfilling the classic conditions for a diagnosis of compulsion. ${ }^{17}$ The desire for a further drink is seen as irrational, the desire is resisted, but the further drink is taken. The patient who is in a withdrawal state (or partial withdrawal) may report that he is compulsively ruminating on alcohol and that he has hit on the strategy of blocking these ruminations by bringing in other lines of thought. It is this feeling of being in the grip of something foreign, irrational, and unwanted which for severely dependent patients seems to be the private experience which is so difficult to convey.

\section{REINSTATEMENT AFTER ABSTINENCE}

Patients usually find abstinence surprisingly easy to maintain, especially in the ward with the usual cues for drinking removed. Later they again begin to drink. Relapse into the previous stage of the dependence syndrome then follows an extremely variable time course. Typically, the patient who had only a moderate degree of dependence will take weeks or months to reinstate it, perhaps pulling back once or twice on the way. A severely dependent patient typically reports that he is again "hooked" within a few days of starting to drink, though even here there are exceptions: on the first day he may become abnormally drunk and be surprised to have lost his tolerance. But within a few days he is again experiencing severe withdrawal symptoms and drinking to relieve them, the subjective experience of compulsivity is reinstated, and his drinking is back in the old, narrowly stereotyped pattern. A syndrome which had taken many years to develop can be fully reinstated within perhaps 72 hours of drinking, and this is one of the most puzzling features of the condition. Studies have shown that a rat given a series of exposures to alcohol will show reinstatement phenomena. $^{\star}$ Reinstatement perhaps in some ways parallels "reminiscence" in a learning theory sense, but there is presumably also a biological contribution.

\section{Syndrome in the round}

Each part of this syndrome relates in some way to each other part, so that to the practised listener the patient's story will describe a particular degree of dependence, shaped and coloured by personality and environment. The patient who has experienced only mild dependence will recount that his drinking repertoire is still varied. Drinking does not yet rule his life. He is not very tolerant to alcohol and may be shocked when he hears how much other patients on the ward have been drinking. He may have experienced withdrawal sweats and occasional retching and will admit that it is sometimes difficult to sign his letters in the morning. He does not take a drink until noon but knows that this drink is important. He is beginning to sense that his control over his drinking is occasionally impaired. He has cut down on his drinking, but it has always slowly built up again. The picture presented by the highly dependent patient is equally coherent, with exactly the same elements but in extreme degree. Assessment of degree of dependence can be made only by careful interpretation of the picture as a whole. When the elements within the story do not add up to a coherent whole most often the doctor has not taken a sufficiently careful history or the patient is withholding some element of the syndrome. Aberrant pictures may also result when the patient is taking drugs or when there is an underlying affective illness or phobic state.

In the definition of any syndrome one question must be whether the cutting point is sharp or blurred-whether the syndrome segments a continuous distribution or whether it represents an abrupt change in distribution. Have many "social drinkers" at some time shown some of the elements of this syndrome in some degree? This type of question will be answered only by further research.

\section{Natural history and social setting}

Natural history is a medical concept-the evolution of a pathological process. The model need not, of course, propose a rigidly stereotyped progression. At present we are ignorant of the variation in natural histories that may be found with the alcohol dependence syndrome. Probably the experience of any degree of dependence carried a threat, but milder degrees can indeed regress and the patient can return to normal drinking. A patient with an intermediate degree of dependence is, if he continues to drink, much more likely to progress to severe dependence than to move backwards down the curve. Very severe dependence is usually irreversible, and if the patient will not accept abstinence he will repeatedly reinstate the syndrome. Even here there may be exceptions. ${ }^{18}$ Little is known about the effect of aging on the syndrome.

What happens to the dependent person is determined not only by the progression of a disease but also by social processes. These help determine the rate of development of the syndrome, the secondary consequencies, the help offered, the degree of stigmatisation. For instance, if alcohol is readily accepted culturally then the picture of dependence may approximate to what Jellinek termed delta alcoholism. If the individual's culture makes a smoothly sustained intake of alcohol difficult to achieve (if he has to observe pub opening hours or gulp down a drink secretly in the cloakroom rather than merge with other drinkers in the cafe) his drinking may be peaky and irregular and of Jellinek's gamma type. ${ }^{1}$ If his culture accepts heavy drinking he may quietly cirrhose his liver without even being put into a deviant role; the same man in a more puritanical culture would find himself cast out of society.

\section{Drink-related disabilities}

A person may, for example, develop cirrhosis, lose his job, crash his car, or break up his marriage through his drinking without suffering from the dependence syndrome. The syndrome should therefore not monopolise medical and social concern. Nevertheless, physical, mental, and social disabilities often accumulate for the person who is 
dependent and are more likely to be incurred the greater his dependence. Greater dependence means both higher alcohol intake and diminished responsiveness to social controls. But the diagnosis of dependence itself and assessment of its degree should be made in relation to the primary symptoms listed at the start of this paper and not by reference to the secondary damage. The analogy is with the classic approach to diagnosis of schizophrenia and the astute recognition of first-rank symptoms.

\section{Looking for the scientific basis}

We are suggesting, then, that a clinical syndrome of alcohol dependence can now be recognised fairly confidently. It is fully in accord with the development of medicine that a syndrome should occasionally be recognised considerably before its scientific basis can be determined. Very speculatively, we may suppose that here the abnormality involves both a biological process and aberrant learning. Reinstatement of tolerance and of withdrawal symptoms suggests that a biologically determined change in response to alcohol may be brought about by certain patterns of exposure. But repeated relief of withdrawal (and of subacute withdrawal), or repeated fear of withdrawal, results in an abnormal drive towards repeated experience of high alcohol intake. The learning process is very incompletely understood, but dependence should perhaps be seen as being in the same group of disorders as phobic and obsessional states, with a potent, complicating, biological factor.

\section{Implication for research and practice}

The presumptive clinical identification of this syndrome has considerable significance for research; one important priority is the sharper delineation of the actual syndrome and of its natural histories and social settings-matters to which the everyday business of clinical observation, as well as more formal research, has much to contribute. Beyond this the research challenge is one of determining piece by piece the psychobiological basis. Better theoretical understanding should open possibilities of more effective treatment, in terms of attack on both biological and learning elements in the pathology.

More immediately an increased awareness of the diagnostic picture and of the need to see dependence in terms of degrees rather than absolutes may have some message for clinical practice. Again using an analogy, alcohol dependence may sometimes have been as loosely diagnosed as schizophrenia, with equally unfortunate consequences. Doctors should be aware that not every patient who drinks too much (for whatever reason) is necessarily dependent on alcohol, and different patients need different help and treatment. There has been some controversy about whether some alcoholics "can return to normal drinking"18 and whether abstinence should still be offered as the only treatment goal; the question may be sharper if applied not just to heterogeneous patient populations but separately to patients with different degrees of dependence. Some preliminary work does indeed suggest that the patients who are less dependent are those more likely to return to controlled drinking. ${ }^{19}$ The clinician who diagnoses moderate or severe dependence might therefore still feel that abstinence is the safest advice. Early dependence becomes a diagnosable condition and is recognisable for the danger which it certainly presents, although the possibility of regression should not be ruled out. This syndrome may therefore have significance both for research and for the clinic and for the fruitful connection between the two.

There may also be a large significance for society as a whole. It should be more widely recognised that our favoured recreational drinks are potentially dependence-inducing drugs. The humane argument that alcoholism should be regarded as a disease seems to have run into difficulties recently because of semantic confusion over what is meant by the over-inclusive term "alcoholism" and the social arbitrariness of the "disease" label. ${ }^{20}$ Without withdrawing sympathy from the non-dependent drinker who is experiencing harm, society should be asked to realise that the person who has become dependent on alcohol is certainly ill; and the possibility of contracting this illness awaits anyone who drinks very heavily.

The ideas expressed in this paper owe much to discussion with colleagues who formed a steering group for a meeting in 1975 of a WHO group of investigators in Geneva; in particular we acknowledge our debt to Dr M Hertzman, Dr M Keller, Mrs J Moser, and Mr $\mathrm{R}$ Room. These international discussions were financially supported by NIAAA (Washington). Responsibility for views expressed is entirely the authors'. Valuable comments on an earlier draft of this paper were given by $\mathrm{Mr}$ A Cartwright, $\mathrm{Dr} \mathrm{D}$ L Davies, $\mathrm{Dr} \mathrm{H}$ Ghodse, Mr S Henry, Dr R Hodgson, Dr R Kumar, Dr G Litman, Dr J Orford, Mr M Raw, Dr B Ridsdale, Dr D Robinson, Dr M Russell, Mr S Shaw, and Dr T Spratley. Mrs Julia Polglaze gave expert secretarial help.

Requests for reprints should be addressed to Dr G Edwards, Addiction Research Unit, Institute of Psychiatry, 101 Denmark Hill, London SE5 8AF.

\section{References}

${ }^{1}$ Jellinek, E M, The Disease Concept of Alcoholism. New Brunswick, Hillhouse, 1960 .

${ }^{2}$ US National Council on Alcoholism, American fournal of Psychiatry, $1972,129,127$.

${ }^{3}$ Edwards, G, Quarterly fournal of Studies on Alcohol, 1974, 35, 176.

4 Brunn, K, et al, Alcohol Control Policies. Helsinki, Finnish Foundation for Alcohol Studies, 1975.

${ }^{5}$ Lelbach, W K, in Recent Advances in Alcohol and Drug Problems I, ed R J Gibbins, p 93. New York, Wiley, 1974.

${ }^{6}$ Meisch, R A, Henningield, J E, and Thompson, T, in Alcohol Intoxication and Withdrawal, ed M M Gross, p 323. New York, Plenum, 1975.

${ }^{7}$ Mello, N K, and Mendelson, J H, Nature, 1965, 206, 43.

${ }^{8}$ Kalant, H, Le Blanc, A E, and Gibbins, R J, in Biological Basis of Alcoholism, ed Y Israel and J Mardones, p 235. New York, Wiley-Interscience, 1971.

${ }^{9} \mathrm{Kalant}, \mathrm{H}$, in Alcohol Intoxication and Withdrawal, ed M M Gross, pp 3-11. New York, Plenum, 1973.

10 Begleiter, $\mathrm{H}$, in Alcohol Intoxication and Withdrawal, ed M M Gross, p 373. New York, Plenum, 1975.

11 Isbell, H, et al, Quarterly fournal of Studies on Alcohol, 1955, 16, 1.

${ }^{12}$ Mendelson, J H, Quarterly fournal of Studies on Alcohol, 1964, suppl 2. Experimentally Induced Chronic Intoxication and Withdrawal in Alcoholics.

${ }^{13}$ Mello, N K, and Mendelson, J H, fournal of Pharmacology and Experimental Therapeutics, 1970, 175, 94.

${ }^{14}$ Gross, M M, and Lewis, E, in Alcohol Intoxication and Withdrawal II, ed M M Gross, p 377. New York, Plenum, 1973.

15 Keller, M, British fournal of Addiction, 1972, 67, 153.

${ }^{16}$ Keller, M, personal communication, 1975.

17 Lewis, A, in Inquiries in Psychiatry, chap 6, p 141. London, Routledge and Kegan Paul, 1967.

18 Davies, D L, Quarterly fournal of Studies on Alcohol, 1962, 23, 94.

19 Orford, J, Behaviour Research and Therapy, 1973, 11, 563.

${ }^{20}$ Robinson, D, Quarterly fournal of Studies on Alcohol, 1972, 33, 1028.

Are workers who deal with phthalic anhydride and naphthalene more likely to develop cancers, including bronchial carcinoma or reticulosis?

Phthalic anhydride, used in manufacturing various phthlates, artificial resins, and benzoic acid, is itself prepared from naphthalene by oxidation. So far there have been no reports of excessive risk of any kind of cancerous disease in men working with either naphthalene or phthalic anhydride. Animal tests have provided no convincing evidence that naphthalene causes cancer in animals exposed to it by a realistic route, although isolated tumours have been seen at the site of its subcutaneous injection in rats and after the introduction of pellets into the lumen of the bladders of mice. Phthalic anhydride does not appear to have been used in formal carcinogenicity tests in animals, perhaps because it resembles no known carcinogen in chemical structure. In general, phthalates have come into prominence recently because their use as plasticisers has led to food becoming contaminated from PVC containers. More seriously, contamination of blood stored in plastic containers with diethylphthalate may have been responsible for hepatitis in patients who have had blood transfusions. Some phthalates are teratogenic. There are no grounds for believing that exposure to phthalic anhydride or naphthalene or both at work increase the risk of developing any form of cancer. 